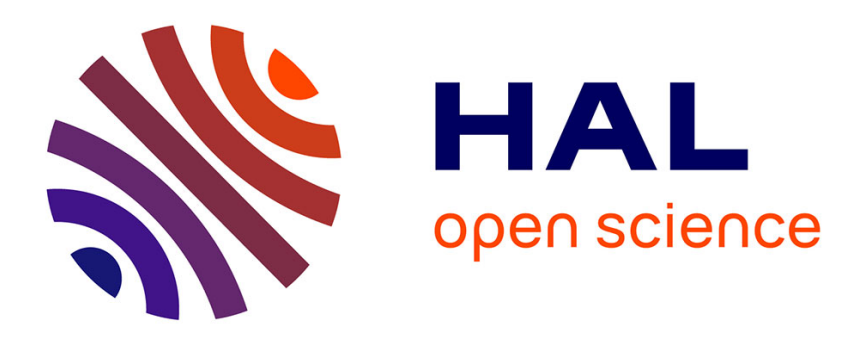

\title{
Étude économique des différentes méthodes d'élaboration du niobium
}

Marcel Vanlaer, Roger Durand

\section{To cite this version:}

Marcel Vanlaer, Roger Durand. Étude économique des différentes méthodes d'élaboration du niobium. Revue de Physique Appliquée, 1970, 5 (3), pp.487-494. 10.1051/rphysap:0197000503048700 . jpa00243419

\section{HAL Id: jpa-00243419 https://hal.science/jpa-00243419}

Submitted on 1 Jan 1970

HAL is a multi-disciplinary open access archive for the deposit and dissemination of scientific research documents, whether they are published or not. The documents may come from teaching and research institutions in France or abroad, or from public or private research centers.
L'archive ouverte pluridisciplinaire HAL, est destinée au dépôt et à la diffusion de documents scientifiques de niveau recherche, publiés ou non, émanant des établissements d'enseignement et de recherche français ou étrangers, des laboratoires publics ou privés. 


\title{
ÉTUDE ÉCONOMIQUE DES DIFFÉRENTES MÉTHODES D'ÉLABORATION DU NIOBIUM
}

\author{
par Marcel VANLAER et Roger DURAND \\ Ugine Kuhlmann, Division Métaux non Ferreux, Brignoud, Isère
}

\begin{abstract}
Résumé. - Par ses propriétés remarquables, le niobium présente un très grand champ d'applications. Chacune d'elles nécessite un métal plus ou moins pur qui, pour une technique donnée, sera plus ou moins cher.

Le procédé utilisé basé sur la réduction métallothermique du pyrochlore suivie d'une purification ou non par distillation des chlorures et terminée par une fusion par bombardement électronique paraît actuellement la plus intéressante. Certes, les prix obtenus sont encore élevés, ceci en raison du faible tonnage élaboré. Par un choix plus judicieux de la qualité en fonction de l'utilisation, on peut espérer augmenter la demande et rendre ce métal aussi compétitif que le zirconium, le molybdène et le tantale, et ce dans un plus grand domaine d'application.
\end{abstract}

\begin{abstract}
Niobium has remarkable properties. It offers a wide range of applications, every of which needs different purities of metal, then different manufacturing processes with different costs.

The most interesting process actually seems to be : pyrochlore's metallothermic reduction, followed or not by chlorides distillation, and finishing with an electron beam melting. That still gives high costs, indeed, because of low quantities which are worked out. We can hope that request will increase, if selecting more judiciously metal's purities in connexion with uses. Then niobium would be done as competitive as zirconium, molybdenium and tantalum, and that would be in a wider range of applications.
\end{abstract}

I. Introduction. - Le niobium est un métal relativement courant dans la croûte terrestre puisque sa concentration atteint le chiffre de $2,4 \times 10^{-3}$ et que les réserves équivalent au total celles du nickel et du molybdène. C'est aussi un métal aux qualités remarquables, que ce soit dans le domaine des hautes températures, de la corrosion, de la transmission des électrons et des neutrons. Ces deux caractéristiques : abondance et propriétés intéressantes devraient suffire à lui valoir une place de choix parmi les éléments métalliques. Or, à part son utilisation massive sous forme de ferro, il est encore d'un emploi très restreint comme métal de base. On ne peut pas dire que sa jeunesse en soit la cause car il est né en même temps que le tantale au siècle dernier, mais il a surtout vécu dans son ombre. En effet, le niobium était un sousproduit de la fabrication du fluotantalate de potassium mise au point par Marignac et, par la suite, il n'était connu que sous une forme particulièrement impure. Mais, depuis la dernière guerre mondiale, d'autres méthodes d'élaboration se sont développées et ont permis de le préparer avec un degré de pureté élevé. Ce n'est pas pour autant que cette industrie se soit développée rapidement.

Nous avons cherché les raisons de cette expansion médiocre et pensé que, outre les difficultés rencontrées dans certaines de ses applications, notamment sa protection contre l'oxydation à haute température, une des causes principales pouvait être son prix.
Nous nous sommes donc attachés à étudier le problème niobium sous l'angle économique. Qui dit prix de revient sous-entend la somme: prix de la matière première + frais de transformation. Or, ces frais de transformation dépendent essentiellement des procédés utilisés et de l'échelle à laquelle ils sont mis en œuvre ; ces deux facteurs sont d'ailleurs euxmêmes fonction de la pureté désirée et des applications que le métal peut recevoir. En effet, plus le métal sera raffiné, plus les frais de transformation seront élevés, et ceci d'autant plus que le débouché sera faible. En définitive, le prix sera lié, outre à la matière première, à la pureté et à la facilité d'écoulement du produit.

C'est surtout sur cette relation du prix avec la pureté que nous insisterons car, si, le plus souvent, le niobium est considéré comme un métal cher, c'est que les utilisateurs exigent une qualité qui n'est pas toujours indispensable à l'application qu'ils veulent en faire et leur hésitation devant un investissement prohibitif se traduit par un renoncement pur et simple. De ce fait, le marché se résorbe, le développement de la production est entravé et l'évolution vers des prix meilleurs est freinée. C'est un cercle vicieux dont on ne peut sortir que par un effort d'information sur la qualité en fonction de l'utilisation. Mais nous parlerons également des nombreuses possibilités que ce métal présente dans des domaines très variés afin d'essayer d'en augmenter le champ d'application. 
Notre plan se trouve ainsi fixé de la façon suivante :

- examen des applications,

- qualité nécessaire à chacune d'elles,

- procédés et minerais à mettre en œuvre pour obtenir la qualité requise,

- prix de revient en fonction des traitements subis et du tonnage,

- comparaison avec les prix des autres métaux afin de savoir dans quelle mesure le métal est compétitif.

II. Applications du niobium. - Le niobium présente des propriétés remarquables : densité relativement faible, température de fusion élevée, environ $2500^{\circ} \mathrm{C}$, excellentes caractéristiques mécaniques à haute température, conductance élevée à basse température, bonne résistance à la corrosion en milieu acide ou en présence de métaux fondus, transparence aux neutrons thermiques, facilité de mise en forme même à froid ; avec le carbone, il forme un carbure très stable; son oxydation ménagée conduit à un film d'oxyde très mince et particulièrement adhérent.

Toutes ces particularités font du niobium, soit à l'état pur, soit allié, un élément de choix dans des domaines très variés :

- celui des aciers sous forme de ferro comme élément stabilisant du carbone des $18 / 8$ à la place du titane ; pour améliorer les propriétés à haute température des aciers du type ferritique utilisés en aéronautique ;

- comme élément de base dans les alliages haute température jusqu'à $1400^{\circ} \mathrm{C}$ à la place des alliages conventionnels limités à $900^{\circ} \mathrm{C}$ et qui sont employés à la confection des réacteurs de fusée et des éléments de guidage des missiles;

- dans l'industrie nucléaire, comme élément de gainage du combustible des breeders.

- en électrotechnique dans la réalisation des alliages supraconducteurs ;

- en électricité dans le domaine des condensateurs de type solide et des redresseurs, les capacités spécifiques du $\mathrm{Nb}$ étant le double de celles du tantale;

- en électronique, comme getter ou émetteur d'électrons ;

- en génie chimique, comme échangeur ou condenseur sur des solutions acides;

- en outre, ses dérivés tels que le carbure entrent dans la fabrication des outils de coupe et son oxyde dans l'industrie des verres optiques et la catalyse d'estérification.

Chacune de ces utilisations nécessite une qualité de métal particulière dont nous allons parler maintenant.

III. Considérations sur la qualité du métal en fonction de son utilisation. - Il est bien évident que la pureté du métal employé dépendra essentiellement de l'utilisation qu'on veut en faire.
- Ainsi, l'aciérie demandera un métal relativement impur, c'est-à-dire en général un ferro-niobium ayant une teneur en $\mathrm{Nb}$ de l'ordre de 60 à $70 \%$; les autres impuretés seront sans importance étant donnée la faible proportion de $\mathrm{Nb}$ dans l'acier (1 à $2 \%$ ). Seul l'étain sera limité à une certaine teneur.

- Dans le cas des alliages réfractaires pour haute température, il ne sera évidemment pas question d'employer du ferro et on tolérera seulement certains métaux réfractaires tels que $\mathrm{Ta}, \mathrm{Mo}, \mathrm{W}$ et même $\mathrm{Zr}$ et $\mathrm{Ti}$. On entre alors dans le domaine du Nb technique de pureté $\geqslant 97 \%$.

- Le génie chimique nécessitera un métal encore plus élaboré et ayant une teneur voisine de $99 \%$ en $(\mathrm{Nb}+\mathrm{Ta})$.

- Les supraconducteurs du type NbTi n'admettent pas plus actuellement de $1500 \mathrm{ppm}$ de Ta, les autres éléments étant inférieurs à $1000 \mathrm{ppm}$, soit environ $99,7 \% \mathrm{Nb}$.

- L'énergie nucléaire exigera moins de 500 ppm de Ta, c'est-à-dire une qualité de niobium à 99,9 \%.

- Enfin, pour des recherches fondamentales, les laboratoires souhaitent un niobium ultra pur, 99,98\%, renfermant moins de $20 \mathrm{ppm} \mathrm{Ta}, 20 \mathrm{ppm} \mathrm{Mo,} 20 \mathrm{ppm}$ W et $100 \mathrm{ppm}$ d'interstitiels. Dans des cas tout à fait exceptionnels, il sera possible de descendre aux $4 \mathrm{~N}$.

En résumé, outre le ferro, on distinguera 3 qualités de $\mathrm{Nb}$ : le technique, le nucléaire et l'ultra pur.

Nous allons voir maintenant comment peuvent être élaborées ces différentes qualités de niobium. Mais d'abord, examinons quels sont les minerais que la nature met à notre disposition.

IV. Sources de niobium. - La composition des principaux minerais de niobium est consignée dans le tableau $n^{0} 1$.

Deux sortes de minerais sont utilisés pour l'obtention du niobium :

- La colombite qui est un niobate de fer et de manganèse contenant de 40 à $75 \%$ de $\mathrm{Nb}_{2} \mathrm{O}_{5}$, une proportion croissante de $\mathrm{Ta}_{2} \mathrm{O}_{5}$ de 2 à $40 \%$ à mesure que la concentration en $\mathrm{Nb}_{2} \mathrm{O}_{5}$ chute, 10 à $20 \%$ de $\mathrm{FeO}$ et de 0 à $10 \% \mathrm{MnO}$, du titane, du W, du Mo et du $\mathrm{Zr}$. En général, ces minerais servent à préparer simultanément le $\mathrm{Nb}$ et le $\mathrm{Ta}$. Les gisements les plus importants se trouvent en Ouganda et au Nigeria où les réserves seraient de $600000 \mathrm{t}$.

- Depuis 1960, un autre minerai, le pyrochlore, a été mis en exploitation au Brésil et au Canada. C'est un fluoniobate de $\mathrm{Ca}$ et $\mathrm{Na}$ contenant de 40 à $65 \%$ de $\mathrm{Nb}_{2} \mathrm{O}_{5}, 10$ à $20 \%$ de $\mathrm{CaO}, 2$ à $6 \%$ de $\mathrm{Na}_{2} \mathrm{O}, 1$ à $10 \%$ de terres rares, du Ti, W, Mo et $\mathrm{Zr}$ et qui présente la caractéristique d'avoir une teneur très faible en tantale de 0,1 à $2 \%$. Il sera donc particulièrement intéressant dans la fabrication du $\mathrm{Nb}$ pur. Les réserves du Brésil seraient de l'ordre de $4 \times 10^{6} \mathrm{t}$. 


\section{Tableau I}

Composition des principaux minerais de niobium

\begin{tabular}{|c|c|c|c|c|c|c|c|c|c|c|c|c|c|c|c|c|}
\hline \multirow{3}{*}{ Minéral } & \multicolumn{16}{|c|}{ Gamme usuelle de concentration $\%$} \\
\hline & $\begin{array}{l}0_{n}^{n} \\
\mathbf{Z}\end{array}$ & 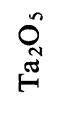 & $\stackrel{0}{3}$ & $\frac{\infty}{0}$ & $\begin{array}{l}\text { O } \\
\text { II }\end{array}$ & ก & $\frac{\text { O্ }}{<}$ & 윽 & $\stackrel{0}{\text { O }}$ & ก๊ & Oี & $\stackrel{\text { ON }}{N}$ & 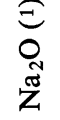 & $\underset{\substack{0 \\
0}}{\overparen{J}}$ & L & 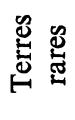 \\
\hline & $\overline{10}$ & $\overline{2}$ & $\overline{0}$ & - & $\overline{10}$ & - & - & - & - & - & $\overline{0}$ & - & 二 & - & 一 & - \\
\hline $\begin{array}{l}\text { NIOBIO-TANTALITE } \\
(\mathrm{Fe}, \mathrm{Mn})(\mathrm{Nb}, \mathrm{Ta})_{2} \mathrm{O}_{6}\end{array}$ & $\begin{array}{c}40 \\
\text { à }\end{array}$ & 2 & 0,2 & 0,2 & 10 & $\operatorname{tr}$ & 0,5 & $\operatorname{tr}$ & 0,5 & 1 & 0,2 & 0,5 & $\operatorname{tr}$ & $\operatorname{tr}$ & - & - \\
\hline Structure : Orthorhombique & 75 & 40 & 2 & 2 & 20 & 1 & 5 & $\operatorname{tr}$ & 10 & 5 & 3 & 6 & 0,5 & 2 & - & - \\
\hline PyROCHLORE & 40 & $\operatorname{tr}$ & $\operatorname{tr}$ & $\operatorname{tr}$ & 2 & 1 & $\operatorname{tr}$ & $\operatorname{tr}$ & $\operatorname{tr}$ & 1 & $\operatorname{tr}$ & 5 & 2 & 10 & 2 & 1 \\
\hline$(\mathrm{Na}, \mathrm{Ca})_{2}(\mathrm{Nb}, \mathrm{Ta})_{2} \mathrm{O}_{6}(\mathrm{OH} \mathrm{F})$ & à & & & & & & & & & & & & & & & \\
\hline Structure : Cubique & 65 & 2 & 1 & 1 & 10 & 8 & 2 & $\operatorname{tr}$ & 1 & 10 & 0,5 & 10 & 6 & 20 & 5 & 10 \\
\hline EUXÉNITE & 4 & 0 & - & - & - & - & - & $\operatorname{tr}$ & - & - & - & 15 & 一 & 0,5 & - & 12 \\
\hline$\left(\mathrm{Y}, \mathrm{Ca}, \mathrm{Ce}, \mathrm{U}, \mathrm{Th}_{1}\right)\left(\mathrm{Nb}, \mathrm{Ta}, \mathrm{Ti}_{2}\right) \mathrm{O}_{6}$ & à & & & & & & & & & & & & & & & \\
\hline Structure : Orthorhombique & 45 & 45 & - & - & - & - & - & $\operatorname{tr}$ & - & - & - & 25 & 一 & 48 & - & 40 \\
\hline ILMÉNORUTILE & $\operatorname{tr}$ & $\operatorname{tr}$ & - & - & 5 & $\operatorname{tr}$ & & $\operatorname{tr}$ & $\operatorname{tr}$ & $\operatorname{tr}$ & $\operatorname{tr}$ & 36 & - & 一 & - & \\
\hline$(\mathrm{Ti}, \mathrm{Fe}, \mathrm{Nb}) \mathrm{O}_{2}$ & à & & & & & & & & & & & & & & & \\
\hline Structure : quadratique & 40 & 35 & - & - & 15 & $\operatorname{tr}$ & - & $\operatorname{tr}$ & - & $\operatorname{tr}$ & 2,5 & 66 & - & - & - & - \\
\hline LOPARITE & & & & & & & & & & & & & 8 & 4 & - & 32 \\
\hline $\begin{array}{l}(\mathrm{Na}, \mathrm{Ce}, \mathrm{Sr}, \mathrm{Ca})(\mathrm{Nb}, \mathrm{Ti}) \mathrm{O}_{3} \\
\text { Structure : Cubique }\end{array}$ & 11,5 & 11,5 & - & - & $\operatorname{tr}$ & $\operatorname{tr}$ & 0,7 & $\operatorname{tr}$ & $\operatorname{tr}$ & 0,5 & - & 39 & $\begin{array}{r}\text { à } \\
10\end{array}$ & 6 & - & 34 \\
\hline
\end{tabular}

$$
\text { (1) } \mathrm{Na}_{2} \mathrm{O}+\mathrm{K}_{2} \mathrm{O} \text {. (2) } \mathrm{CaO}+\mathrm{SrO}+\mathrm{BaO}
$$

- Une source accessoire est constituée par les scories de traitement de la cassitérite qui contiennent environ $14 \% \mathrm{Nb}_{2} \mathrm{O}_{5}$ et moins de $3 \%$ de $\mathrm{Ta}_{2} \mathrm{O}_{5}$.

- Enfin, d'autres minerais contenant du $\mathrm{Nb}$ et du Ta, tels que l'euxénite, l'ilménorutile, la loparite, sont traités pour la récupération d'autres métaux; titane, uranium, thorium, rhénium, et même le tantale dont les ressources sont relativement faibles.

En dehors des scories de cassitérite, tous ces minerais font l'objet d'une concentration sur le carreau de la mine soit par lévigation, triage magnétique ou électrostatique, flottation, car il n'est pas rare d'exploiter des gisements dits économiques alors que la concontration de la roche ne dépasse pas $0,1 \%$ de $\mathrm{Nb}_{2} \mathrm{O}_{5}$.

Ceci permettra de comprendre pourquoi les prix des minerais sont très élevés, de l'ordre de $16 \mathrm{~F} / \mathrm{kg} \mathrm{Nb}$ alors que pour le $\mathrm{Ta}$, ces chiffres atteignent presque $100 \mathrm{~F} / \mathrm{kg}$.

Quels vont être les traitements subis par ces différents minerais en vue d'obtenir les qualités de niobium énumérées précédemment ? C'est ce que nous allons examiner maintenant.

V. Elaboration du niobium. - Nous nous limiterons à l'emploi du pyrochlore car c'est à la fois la matière première la plus répandue, la plus concentrée en $\mathrm{Nb}$ et la moins chargée en tantale. Dans ce minerai, le niobium est associé à de nombreuses impuretés qui peuvent se classer en trois groupes :

- les alcalins, alcalinoterreux, métalloïdes et terres rares,
- les métaux moins réfractaires que le $\mathrm{Nb}$ : $\mathrm{Al}, \mathrm{Zr}, \mathrm{Hf}, \mathrm{Ti}, \mathrm{Fe}, \mathrm{Ni}, \mathrm{Mn}, \mathrm{Si}, \mathrm{Sn}$,

- les métaux plus réfractaires : Mo, W, Ta, avec une mention particulière pour ce dernier qui a même structure électronique et même rayon atomique que $\mathrm{Nb}$, donc des propriétés chimiques et physiques voisines, ce qui laisse supposer une séparation difficile.

Le cycle d'élaboration du métal jusqu'à l'état lingot comporte, en général, trois étapes :

- extraction du $\mathrm{Nb}$ sous forme d'un composé pur (séparation des impuretés métalliques),

- réduction de ce composé en métal brut,

- consolidation et affinage en interstitiels.

Nous allons retracer la voie qui a été suivie pour parvenir à chacune des trois qualités de niobium : technique, nucléaire et ultra pur.

L'ensemble des procédés mis en œuvre figure dans le tableau $n^{\circ}$ II.

1. OBTENTION DU NIOBIUM TECHNIQUE. - Elle consiste en une réduction directe du pyrochlore par métallothermie suivie d'une purification par fusion au four à bombardement électronique. La première étape d'extraction est donc by-passée. Au cours de la réduction se produit une élimination des terres rares, des alcalins, alcalino-terreux et une partie du zirconium. Il reste dans le métal brut obtenu des interstitiels : $\mathrm{C}, \mathrm{N}_{2}, \mathrm{O}_{2}$, des éléments volatifs : $\mathrm{Al}, \mathrm{Si}, \mathrm{Fe}$, $\mathrm{Ti}, \mathrm{Zr}$, des éléments réfractaires : W, Mo, Ta. 
Tableau II

Schéma de fabrication du niobium

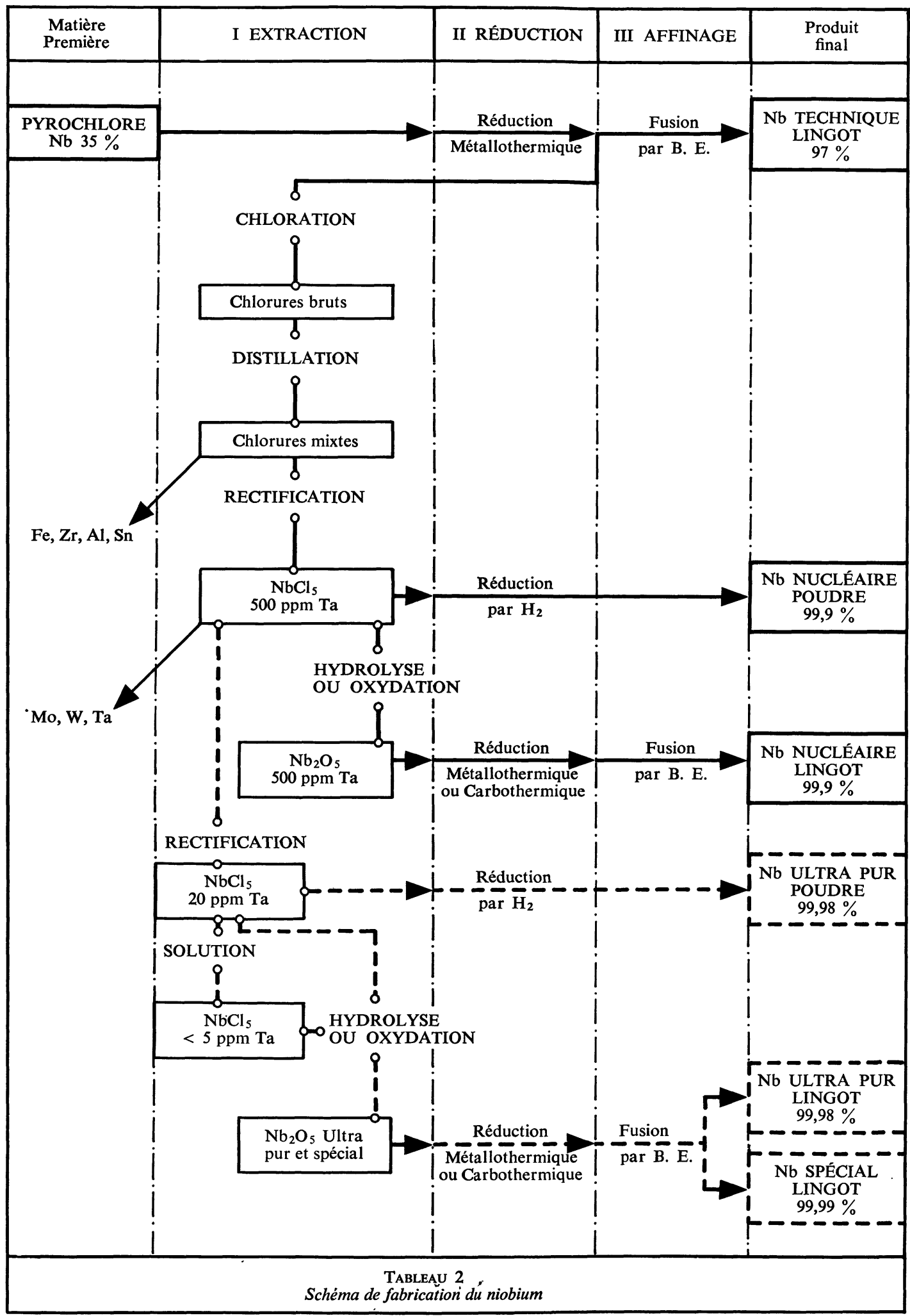


La fusion par bombardement électronique (cf. Fig. 1 et 2) permet d'éliminer partiellement les deux premiers groupes d'impuretés sous forme élémentaire pour $\mathrm{Fe}, \mathrm{Ti}, \mathrm{N}_{2}$, de sous-oxyde pour $\mathrm{Al}, \mathrm{Si}, \mathrm{C}$;

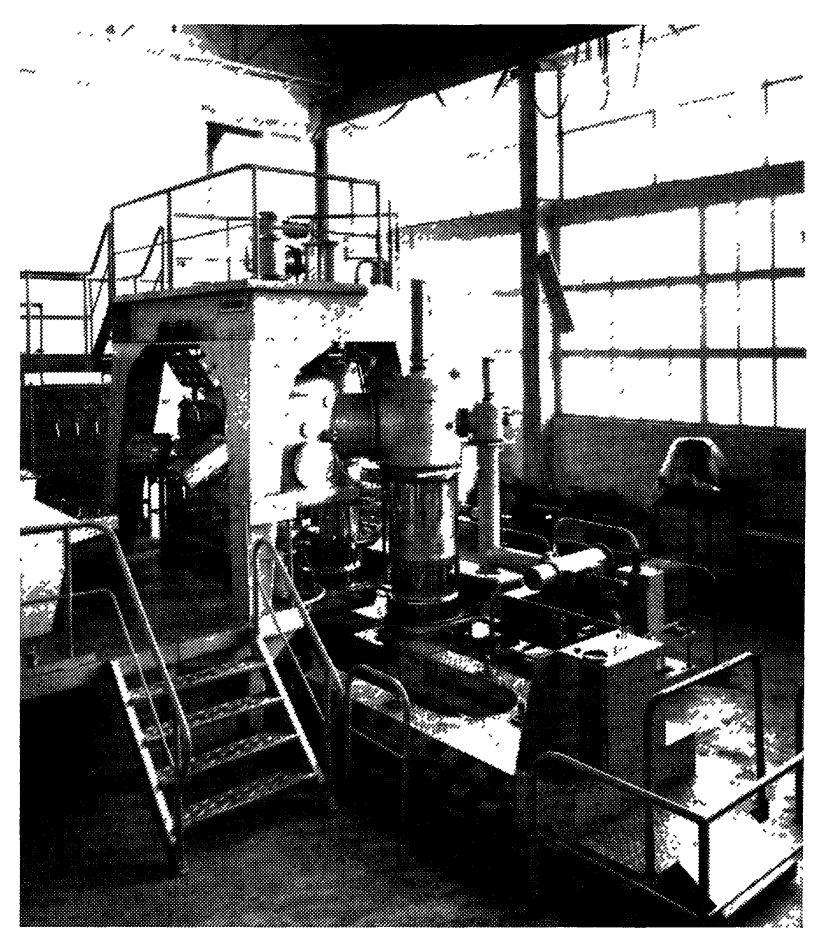

Fig. 1. - Vue générale du four à bombardement électronique de $200 \mathrm{~kW}$.

une quantité appréciable d'oxygène s'en va à l'état de $\mathrm{NbO}$, quant aux éléments réfractaires $\mathrm{Ta}, \mathrm{Mo}, \mathrm{W}$, ils se concentrent.

On retrouve donc un métal plus ou moins riche en Ta, Mo, W, suivant la teneur du minerai en ces impuretés. En moyenne, Ta sera voisin de 1,5\%, Mo 0,5\% et W $0,5 \%$. En somme, le niobium obtenu titre environ $97 \%$.

2. ObTENTION DE NIOBIUM NUClÉAIRE. - Le métal subit dans ce cas les trois étapes d'élaboration, c'està-dire extraction, réduction et affinage.

$\mathrm{Au}$ cours de l'extraction, le pyrochlore est réduit par métallothermie puis chloré. L'ensemble des chlorures solides à température ordinaire, mais gazeux et stables au-dessous de $350^{\circ} \mathrm{C}$, peut être soumis à une rectification fractionnée. Les températures d'ébullition des chlorures de $\mathrm{Si}, \mathrm{Fe}, \mathrm{Ti}, \mathrm{Mo}, \mathrm{W}$,

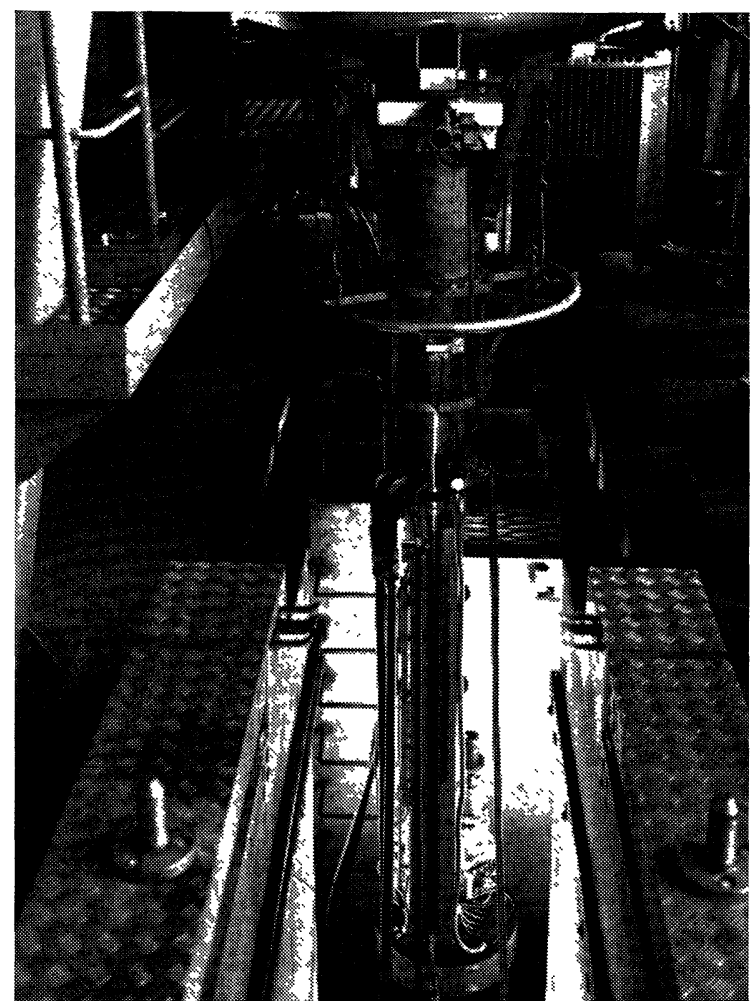

FIG. 2. - Un « extracteur» du four à bombardement électronique (lingotière sous vide de $2 \times 10^{-5}$ Torr).

$\mathrm{Zr}$ et $\mathrm{Nb}$, sont suffisamment différentes pour permettre une excellente séparation.

Seul le chlorure de tantale bouillant à $235^{\circ} \mathrm{C}$, alors que le $\mathrm{NbCl}_{5}$ bout à $248^{\circ} \mathrm{C}$, est assez difficile à éliminer. Il faut employer des colonnes de rectification ayant un grand nombre de plateaux théoriques et un taux de reflux suffisamment élevé pour recueillir un $\mathrm{NbCl}_{5}$ contenant moins de 500 ppm de Ta (cf. Fig. 3).

Par la suite, ce chlorure peut être réduit directement par l'hydrogène pour obtenir de la poudre (cf. Fig. 4), soit transformé en oxyde, réduit par métallothermie ou carbothermie (cf. Fig. 5) et fondu par bombardement électronique.

$\mathrm{Au}$ cours de la fusion, on se retrouvera dans les conditions précédentes avec cependant nettement moins de fer, aluminium, silicium, tantale, molybdène, tungstène. Le tableau no III montre d'ailleurs une analyse du produit brut, ainsi que l'évolution des différentes impuretés au cours de 3 fusions successives.

TABLEAU III

Affinage du niobium par bombardement électronique

\begin{tabular}{|c|c|c|c|c|c|c|}
\hline \multicolumn{6}{|c|}{ Eléments $\times 10^{-6}$} & \multirow{2}{*}{$\begin{array}{c}\text { Dureté } \\
\text { HV } 30\end{array}$} \\
\hline Qualité du métal & $\mathrm{O}$ & $\mathrm{C}$ & $\mathbf{N}$ & $\mathrm{Si}$ & $\mathrm{Fe}$ & \\
\hline - & 一 & 一 & - & 一 & 一 & - \\
\hline um brut & 18200 & 590 & 850 & 2000 & 5000 & \\
\hline ès $1^{\text {re }}$ fusion & 1380 & 16 à 32 & 261 & 102 & 100 & 150 à 170 \\
\hline $2^{e}$ fusion & 575 & 8 à 22 & 91 & 27 & 35 & 80 à 100 \\
\hline $3^{\mathrm{e}}$ fusion $\ldots \ldots$ & 175 & 6 à 14 & 39 & 8 & $<20$ & 60 à 70 \\
\hline
\end{tabular}




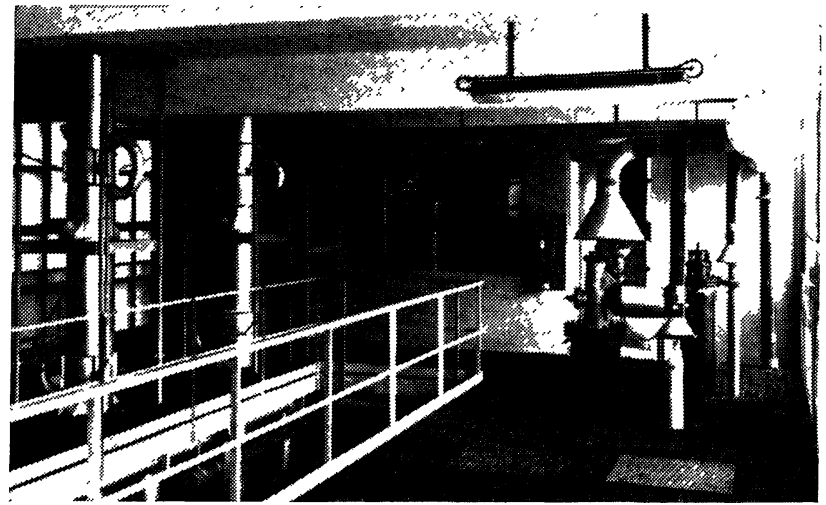

Fig. 3. - Rectification fractionnée du chlorure de Niobium : Vue partielle des colonnes de distillation de l'Atelier industriel.

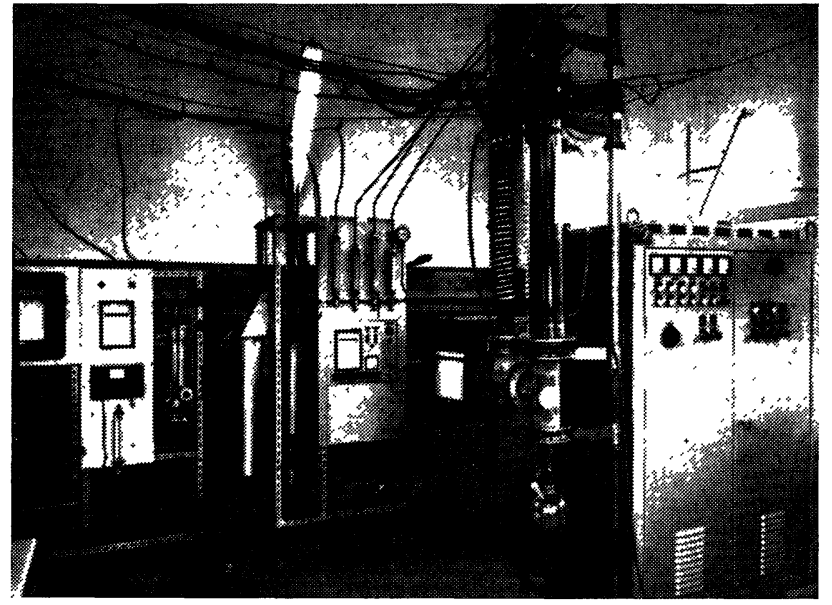

Fig. 4. - Chaîne pilote de production du Niobium "poudre» par réduction du chlorure par l'hydrogène.

Il se produit une élimination rapide de tous les éléments métalliques ou métalloïdes, sauf l'oxygène dont la diminution nécessite plusieurs fusions. Néanmoins, après 3 passages, cette teneur est réduite à moins de 200 ppm ce qui, compte tenu du tantale, conduit finalement à un niobium à 99,9\%

Le métal présente alors une dureté HVN 30 de 60 à 70 , ce qui le rend particulièrement apte à toute transformation.

3. ObTENTION D'UN NIOBIUM ULTRA PUR. - Le processus de fabrication est semblable à celui du

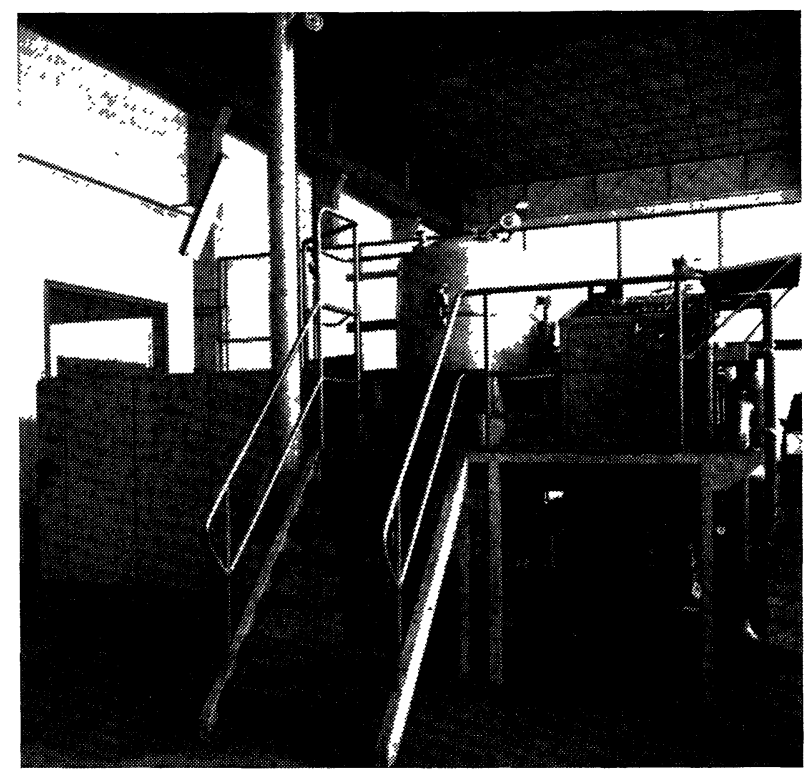

Fig. 5. - Réduction carbothermique de l'oxyde de Niobium. Vue d'un four industriel.

niobium nucléaire, avec toutefois une purification en tantale plus poussée : les fractions titrant 500 ppm subissent un recyclage dans une nouvelle rectification, ce qui permet de descendre à des concentrations inférieures à $20 \mathrm{ppm}$ et aussi en sélectionnant certaines fractions de queue, on peut arriver au-dessous de 5 ppm.

D'autre part, au cours de l'affinage, le nombre de fusions passe de 3 à 6 , de façon à réduire la quantité d'oxygène aux environs de $30 \mathrm{ppm}$.

Aussi obtient-on un niobium à $99,98 \%$ et même à quatre neuf en prenant certaines précautions.

4. Conclusions. - Il apparaît donc que les trois méthodes d'élaboration sont basées principalement sur une extraction plus poussée du tantale, comme en témoigne le tableau d'analyse $\mathrm{n}^{\circ} \mathrm{IV}$, La technique de fabrication exploitée par UGINE KUHLMANN est particulièrement apte à produire économiquement les trois qualités de niobium. En effet, le procédé mettant en œuvre la rectification des chlorures produit intermédiairement les qualités technique et nucléaire quand on passe du minerai au niobium ultra pur.

TABLEAU IV

Analyse des différentes qualités de niobium

\begin{tabular}{|c|c|c|c|c|c|c|c|c|c|}
\hline Qualité & Pureté & \multicolumn{8}{|c|}{ Eléments $\times 10^{-6}$} \\
\hline du métal & - & C & $\underline{\mathrm{O}_{2}}$ & $\underline{\mathrm{N}_{2}}$ & $\mathrm{Ta}$ & Mo & W & $\mathrm{Fe}+\underline{\mathrm{Ni}}+\mathrm{Cr}$ & $\mathrm{Zr}+\mathrm{Ti}$ \\
\hline $\begin{array}{l}\text { Niobium } \\
\text { technique }\end{array}$ & $>97$ & $<1000$ & $<200$ & $<200$ & $1,5 \%$ & $<0,5 \%$ & $<0,5 \%$ & $<500$ & $<1000$ \\
\hline $\begin{array}{l}\text { Niobium } \\
\text { lucléaire }\end{array}$ & $>99,9$ & $<100$ & $<200$ & $<100$ & $<500$ & $<20$ & $<20$ & $<20$ & $<20$ \\
\hline Niobium & $>99,98$ & $<30$ & $<30$ & $<30$ & $<20$ & $<20$ & $<20$ & $<20$ & $<20$ \\
\hline
\end{tabular}

ultra pur 
VI. Considérations sur les prix de revient en fonction de la qualité et du tonnage. - Nous avons vu qu'en fonction de la qualité désirée, les méthodes d'élaboration étaient plus ou moins compliquées et, par suite, coûteuses. A la lumière de notre expérience, nous avons chiffré les différents prix auxquels on arrivait dans chacune des trois qualités pour une production de quelques tonnes par an et pour un métal livré sous forme de tôles de $1 \mathrm{~mm}$ d'épaisseur. Deux chiffres ont été avancés : le prix au kilogramme et celui au $\mathrm{m}^{2}$; nous verrons en effet l'intérêt de ce dernier dans la comparaison avec les autres métaux.

L'obtention du niobium technique nécessite deux étapes de procédés : la réduction et l'affinage, suivies évidemment des opérations de forgeage et de laminage : c'est la voie la plus courte et la moins chère car la réduction nécessite un matériel assez rudimentaire et l'affinage met en œuvre le four à bombardement d'électrons pour un nombre de fusions limité. De ce fait, les chiffres obtenus se situent aux environs de $2300 \mathrm{~F}$ le $\mathrm{m}^{2}$; étant donnée l'évolution actuelle du problème et les progrès en voie de réalisation, nous envisageons une diminution sensible de ce prix au cours des prochaines années pour atteindre $1600 \mathrm{~F} / \mathrm{m}^{2}$. Dans l'hypothèse d'une production de plusieurs dizaines de tonnes par an, ce prix pourrait être facilement divisé par un facteur 2.

En ce qui concerne le niobium nucléaire, la technique d'obtention est beaucoup plus évoluée. Elle nécessite une étape d'extraction assez complexe passant par les stades de chloration, de rectification et d'oxydation, opérateurs qui grèvent lourdement le prix de revient. Par la suite, l'étape affinage est également plus poussée. Ainsi, la valeur du métal obtenue sera sensiblement le double de celle du métal technique, c'est-à-dire environ $5100 \mathrm{~F} / \mathrm{m}^{2}$. Ici encore le tonnage jouera son rôle et un débouché de 50 à 100 tonnes/an permettrait de réduire ce prix aux environs de $3000 \mathrm{~F} / \mathrm{m}^{2}$.

Dans le domaine de l'ultra pur, chacun sait que les dernières ppm d'impuretés sont les plus difficiles à enlever, aussi faudra-t-il recourir à une extraction fine par rectifications successives et à l'affinage poussé par fusions multiples et prendre de multiples précautions pour éviter toute pollution lors de la transformation en tôle. Par ailleurs, comme il s'agit d'une élaboration à l'échelle pharmaceutique, le prix sera forcément très élevé : $5500 \mathrm{~F} / \mathrm{kg}$ et $45000 \mathrm{~F} / \mathrm{m}^{2}$. Cependant, ce prix n'a rien de prohibitif si on le compare à celui pratiqué aux Etats-Unis et qui, pour un niobium à $99,98 \%$, répondant à l'analyse suivante : $\mathrm{O}_{2} 20 \mathrm{ppm} ; \mathrm{C} 15 \mathrm{ppm} ; \mathrm{N}_{2} 15 \mathrm{ppm} ; \mathrm{H}_{2} 1 \mathrm{ppm}$; Ta 50 ppm ; Fe 15 ppm ; Si 15 ppm ; Cr 10 ppm ; Mo 50 ppm ;

s'élève à $11000 \mathrm{~F} / \mathrm{kg}$ pour du rond de 10 et $20000 \mathrm{~F} / \mathrm{kg}$ pour de la tôle, soit au $\mathrm{m}^{2}: 171400 \mathrm{~F}$.

VII. Comparaison des prix du niobium et des autres métaux et alliages réfractaires. - Nous avons rassemblé également dans le tableau no $\mathrm{V}$ les prix des diffé-

\section{TABLEAU V}

Tableau de comparaison de prix du niobium et des autres métaux et alliages réfractaires

\begin{tabular}{|c|c|c|c|c|}
\hline & & & Tôle 1 & aisseur \\
\hline & & & $>1 \mathrm{t}$ & $\operatorname{Prix} / \mathrm{m}^{2}$ \\
\hline Métal & Nuance & Densité & Prix/kg & - \\
\hline A . & $100-$ & $\overline{-1}$ & F & \\
\hline Acier inoxydable $\ldots$. & $18 \mathrm{Cr} 10 \mathrm{Ni}$ & 7,99 & 6 & 48 \\
\hline Acier réfractaire ......, & $25 \mathrm{Cr} 20 \mathrm{Ni}$ (NS 30) & 7,98 & 11 & 81,5 \\
\hline & $\begin{array}{l}\text { 1,5 Cr } 25 \mathrm{Ni} 1,2 \mathrm{Mo} \\
2 \mathrm{Ti} \text { (ATVS) }\end{array}$ & 一 & 28 & 225 \\
\hline$\ldots \ldots \ldots$ & Pur & 8,9 & 44 & 400 \\
\hline Superalliage $\ldots \ldots \ldots$ & $20 \mathrm{Cr} 10 \mathrm{Ni} 15 \mathrm{~W}$ (ATGH) & 9,25 & 68 & 545 \\
\hline & $\begin{array}{l}\text { Hastelloy B (63 Ni } 28 \mathrm{Mo} 1 \mathrm{Cr} \\
6 \mathrm{Fe} 1 \mathrm{Mn} 1 \mathrm{Si})\end{array}$ & - & 106 & 980 \\
\hline Titane & pur & 4,54 & 80 & 360 \\
\hline Zirconium ......... & nucléaire pur & 6,5 & 185 & 1200 \\
\hline & commercial pur & 6,5 & 135 & 880 \\
\hline Molybdène & pur & 10,2 & 384 & 3916 \\
\hline Tantale ...... & pur & 16,6 & 650 & 10800 \\
\hline & ultra pur 99,98 & 8,57 & 5500 & 45000 \\
\hline & nucléaire (en 1969) & - & 600 & 5100 \\
\hline Niobium ..... & nucléaire (dans avenir) & - & 350 & 3000 \\
\hline & technique (en 1970) & 一 & 270 & 2300 \\
\hline & technique (dans avenir) & - & 190 & 1600 \\
\hline & $\begin{array}{l}\text { technique (production très } \\
\text { importante) }\end{array}$ & - & 100 & 850 \\
\hline
\end{tabular}


rents métaux et alliages réfractaires en partant des inoxydables classiques (type 18/8), en passant par les aciers réfractaires (type 25/20 au molybdène), le nickel pur, les superalliages à base nickel, le titane, le zirconium, le molybdène et le tantale.

Ces prix ont été établis, comme pour le niobium, à la fois au $\mathrm{kg}$ et au $\mathrm{m}^{2}$ pour des tôles de $1 \mathrm{~mm}$ d'épaisseur livrées par quantité minium de $1 \mathrm{t}$.

Evidemment, les chiffres au $\mathrm{m}^{2}$ sont les plus significatifs car, dans toutes les applications, on s'intéressera davantage à la surface de tôle qu'on peut obtenir pour une somme donnée qu'au poids. Ainsi, par exemple, dans notre tableau on trouvera pour le tantale pur et le niobium de qualité nucléaire, des prix au $\mathrm{kg}$ identiques ; en fait, en raison d'un rapport de densité égal à 2 , il sera plus intéressant de prendre du niobium car pour construire le même appareil, l'investissement sera moitié de celui nécessité par le tantale.

Ce tableau montre une gradation croissante des prix au $\mathrm{m}^{2}$ en passant des aciers inox $18 / 10$ à $48 \mathrm{~F}$ aux aciers réfractaires de 81 à $225 \mathrm{~F}$, au titane à $360 \mathrm{~F}$, au nickel à $400 \mathrm{~F}$, aux superalliages de 545 à $980 \mathrm{~F}$, au zirconium de 880 à $1200 \mathrm{~F}$ suivant qu'il est hafnié ou non, au molybdène à $3900 \mathrm{~F}$, jusqu'au tantale à $10800 \mathrm{~F}$. Quant au niobium, suivant sa qualité, il se situe actuellement, pour le technique : entre le zirconium et le molybdène, pour le nucléaire : entre le molybdène et le tantale, et l'ultra pur défie évidemment toute concurrence.

Mais, dans la perspective de tonnage plus impor- tant, on s'aperçoit que le nucléaire sera moins cher que le molybdène et que le technique chutera à un prix voisin de celui du zirconium.

Certes, ces comparaisons peuvent paraître arbitraires car chaque métal ayant des propriétés bien particulières et un champ d'application limite, il est bien souvent impossible de faire un choix en fonction du prix. Mais elles ont l'avantage de montrer que le niobium est somme toute un métal d'un prix abordable et d'autant plus que ses débouchés se développeront.

VIII. Conclusions. - Dans ce court exposé, nous avons essayé de montrer qu'en raison de l'abondance de ses minerais et de ses propriétés remarquables, le niobium pouvait être une solution à de nombreux problèmes posés par les techniques les plus modernes. Si son utilisation est encore réduite, c'est qu'il a la réputation d'être un métal cher. En fait, il faut tenir compte de la pureté qui met en jeu des méthodes de préparation plus ou moins élaborées et des débouchés offerts aux différentes qualités obtenues. Dans la gamme des produits élaborés par UGINE KUHLMANN, il apparaît que le niobium supporte la comparaison avec d'autres métaux réfractaires ayant un éventail de possibilités beaucoup moins large. Mais il est bien certain qu'il ne deviendra vraiment compétitif que dans la mesure où de larges débouchés lui seront assurés.

Il appartient à chaque technicien de penser niobium afin que ce métal ne reste plus un métal d'avenir mais devienne un métal du présent.

\section{QUESTIONS ET RÉPONSES FORMULÉES EN SÉANCE}

M. X.... - Quelle est la consommation actuelle des Etats-Unis en Niobium pur?

M. VANLAER. - Elle a été de $45 \mathrm{t}$ en Niobium pur en 1964 alors que la production sous forme de ferroniobium était 10 fois plus importante.

M. CRussard. - L'écart de pureté, entre les qualités: technique et nucléaire, est très important $(97 \%$ et $99,9 \%$ ), ne serait-il pas préférable d'avoir des teneurs intermédiaires?

M. VANLAer. - Les chiffres donnés ne sont que des valeurs particulières de pureté. Notre technique par rectification des chlorures présente l'intérêt de pouvoir fournir toutes les qualités intermédiaires par une rectification plus ou moins poussée.
M. X.... - Quel est le prix actuel du chlorure de niobium?

M. VANLAer. - Environ $150 \mathrm{~F} / \mathrm{kg}$ de niobium contenu dans le chlorure de qualité nucléaire.

M. BRUnetaud. - Quel est le prix des alliages de niobium ?

MM. VANLaer et Durand. - Les quantités produites actuellement sont trop faibles pour donner un prix valable. En fait, le prix des alliages dépend:

- du prix du niobium qui est fonction, comme nous l'avons montré, de la qualité et de la quantité,

- du prix de la transformation, donc des moyens à mettre en œuvre et de la mise au mille. Pour une production à l'échelle industrielle, ces frais doivent être comparables à ceux de la transformation des superalliages. 